\title{
Vannes cylindriques de protection des turbomachines hydrauliques
}

\section{Cylindrical protection valves for hydraulic turbine machinery}

\author{
S. Casacci ${ }^{1}$, P. Hudon ${ }^{2}$ et J. Bosc ${ }^{1}$ \\ (1) Neyrpic, Grenoble \\ (2) Marine Industrie Limitée, Sorel, \\ Québec, Canada
}

\section{Introduction}

La recherche de la meilleure rentabilité globale des installations hydroélectriques peut se rapporter aux organes de protection des turbomachines hydrauliques, dont la conception et la disposition ont une répercussion sensible sur le coût des aménagements. Une économie importante peut être réalisée en supprimant la vanne de pied et en conservant une vanne de tête commandée automatiquement. Cette disposition a été retenue en particulier pour les centrales de Churchill Falls (Canada) : 11 groupes de $475 \mathrm{MW}$ sous $320 \mathrm{~m}$ de chute, et d'Alcantara (Espagne): 4 groupes de $242 \mathrm{MW}$ sous $97 \mathrm{~m}$ de chute. Elle présente quelques inconvénients. $\mathrm{Si}$, lors des arrêts, on baisse la vanne de tête, la conduite se vide à travers le distributeur et il faut la remplir avant le démarrage suivant. D'autre part, notamment pour les hautes chutes, les cycles de charge et de décharge appliqués à la conduite ne sont pas sans effet sur elle et sur son blocage dans le rocher. Si, au contraire, on la maintient ouverte, les fuites sous la pression maximale entraînent des pertes d'énergie et des usures par cavitation à l'aval des directrices. Par ailleurs, l'exploitation des turbomachines pour la production d'énergie de pointe nécessite des temps de démarrage très courts, incompatibles avec la durée de remplissage d'une conduite. Il faut donc revenir à une vanne de pied étanche.

Les solutions classiques: robinets papillon et robinets sphériques restent relativement coûteuses; l'installation de ces matériels dans l'aménagement entraine une plusvalue sur le Génie civil car ils nécessitent une implantation soit dans des fosses qui augmentent la largeur de la centrale, soit dans une galerie séparée. Au contraire, la vanne fourreau, tout en assurant la protection et l'étanchéité à un prix moindre, a peu d'impact sur les dimensions de la centrale en raison de son emplacement et de son faible encombrement. En outre, aspect qu'il ne faut pas négliger de nos jours, la vanne fourreau ne cause pas de pertes de rendement, ce qui n'est pas le cas des vannes papillon. Pour la centrale de $L G \cdot 2$, par exemple, la présence de telles vannes dans les conduits d'amenée aurait pu entraîner des pertes annuelles de cent millions de kWh.

Sur un autre plan, lors des coupures du débit par une vanne fourreau, du fait de l'axisymétrie des écoulements, les régimes perturbés sont moins violents que pour des robinets classiques; les structures fixes et tournantes se trouvent soumises à des fluctuations d'effort de moindre amplitude. Ce dernier aspect revêt une importance particulière pour certaines turbines-pompes.

L'expérience industrielle de la firme Neyrpic et de sa licenciée canadienne, Marine Industrie Limitée (MIL), concernant la conception et la réalisation des vannes cylindriques permet actuellement de faire le point sur l'intérêt de ce matériel.

\section{Premières réalisations}

Le premier brevet de vanne fourreau déposé par Neyrpic remonte à 1947 . Les premières réalisations ont été pour la centrale de Monteynard, comportant 4 groupes de $83 \mathrm{MW}$ sous $127 \mathrm{~m}$ de chute et dont la première mise en service a été faite en 1962. Dans le but de réduire la largeur de la salle des machines, logée dans le cœur même du barrage, des vannes fourreau ont été adoptées pour assurer l'étanchéité. Ces vannes, dont le diamètre intérieur est de $3410 \mathrm{~mm}$, l'épaisseur $75 \mathrm{~mm}$ et la hauteur de $980 \mathrm{~mm}$, sont placées entre les directrices et la roue. Elles n'ont pas été prévues pour couper le débit de la turbine. Chaque vanne est suspendue en trois points à $120^{\circ}$ à des tiges filetées verticales manœuvrées par trois vérins électriques comprenant chacun un réducteur de vitesse d'angle, entraîné par un moteur électrique à frein incorporé avec un accouplement à limiteur de couple. Un dispositif à selsyns différentiels assure la synchronisation entre les trois vérins d'entraînement (voir Fig. 1) 


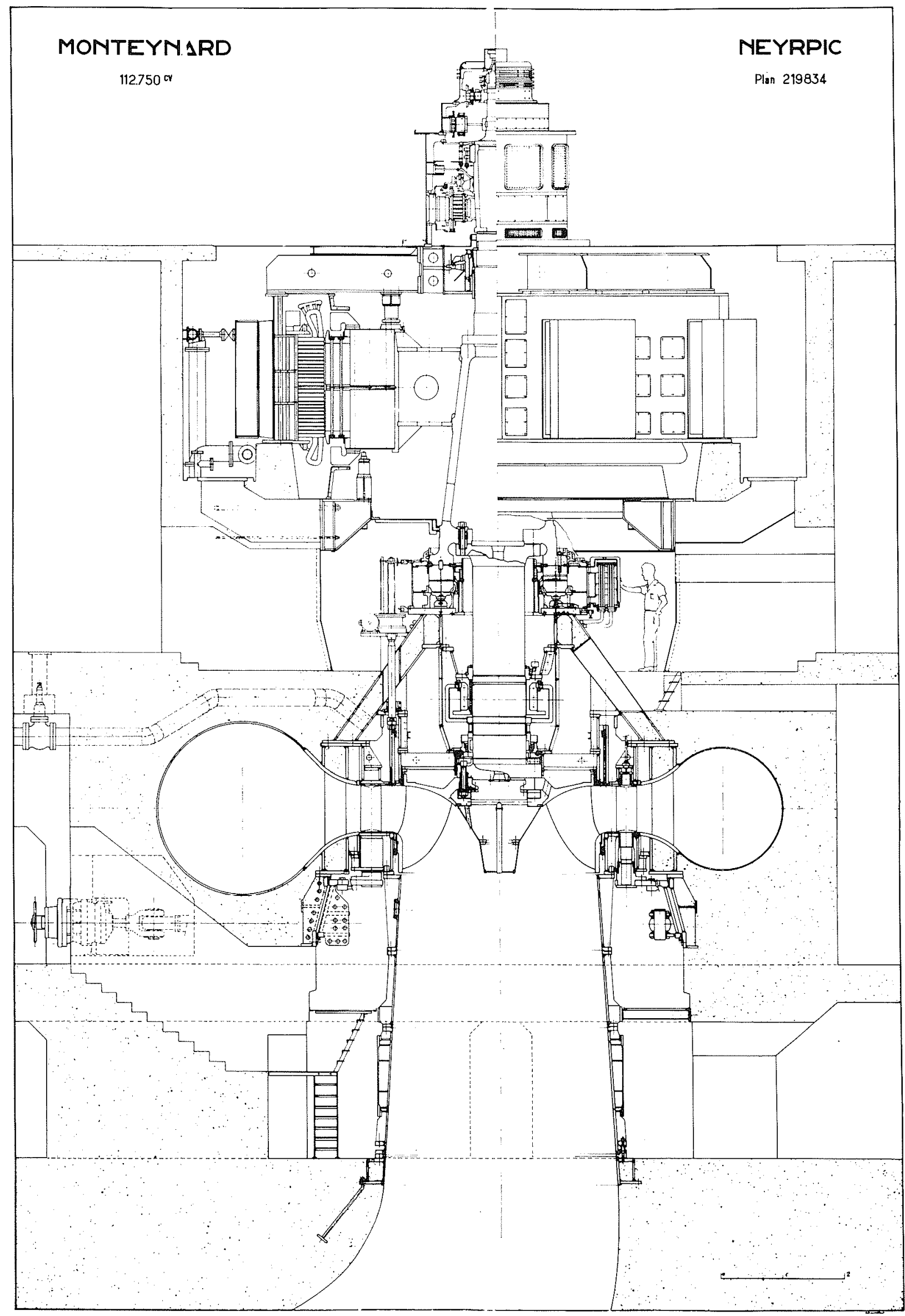

Figure 1 - Ensemble coupe d'un groupe de Monteynard muni d'une vanne cylindrique 
La vanne fourreau est soulevée en position ouverte. Elle se loge alors dans une chambre qu'il faut décomprimer avant chaque mancuvre d'ouverture et de fermeture.

L'étanchéité en position fermée est assurée à la partie basse par un joint caoutchouc en forme de $T$ fixé sur la tranche inférieure de la vanne et venant s'appuyer sur le flasque inférieur et à la partie supérieure par un joint en note de musique placé sur le diamètre intérieur de la virole cylindrique s'appuyant sur le flasque supérieur.

A la centrale de Teillet Argenty la vanne fourreau est prévue pour couper le débit maximal en cas de défaillance du vannage. Elle est installée sur un groupe de $16,4 \mathrm{MW}$ sous 41,7 m de chute mis en service en 1965 . Elle se loge entre l'avant-distributeur et le distributeur, le guidage étant assuré par appui direct avec jeu sur les dix avant-directrices. Elle est constituée d'une virole cylindrique de diamètre intérieur $3350 \mathrm{~mm}$ et d'épaisseur $30 \mathrm{~mm}$. La hauteur du distributeur est de $746 \mathrm{~mm}$.

La commande de la vanne se fait par trois vérins verticaux à huile situés au-dessous de la vanne qui est baissée en position ouverte. L'un des vérins sert de pilote et les deux autres lui sont asservis hydrauliquement.

L'étanchéité en position fermée est assurée par trois joints en note de musique : l'un d'eux est disposé sur la tranche supérieure de la vanne, les deux autres étant situés à la partie inférieure respectivement sur les diamètres intérieur et extérieur.

A la mise en service, on a constaté des blocages de la vanne surtout en cours de fermeture. Ces blocages ont été attribués essentiellement au dispositif de commande par un vérin pilote, les vérins pilotés prenant du retard sur le vérin pilote. Les défauts à la fermeture ont cessé après que le dispositif de commande fut modifié complètement au profit de la synchronisation des 3 vérins par l'admission d'un même volume d'huile dans chacun d'eux.

Outre les centrales de Monteynard et de Teillet Argenty, des vannes fourreau ont également été adoptées par EDF dans quatre autres installations en France: Besserve, Mont-Larron, Saint-Georges de Commiers, Le Teich.

Parallèlement à l'utilisation en France, les vannes fourreau ont été employées également au Canada.

\section{Nouvelles réalisations}

C'est forte de l'expérience acquise par Hydro-Québec aux centrales d'outardes 3 et de Manic 3, et qui en ont fait un pionnier dans l'utilisation des vannes cylindriques de grandes dimensions, que la Société d'Energie de la Baie James (SEBJ) a choisi ce type de vanne pour $L G-2$, la plus puissante centrale hydroélectrique au Canada.

Les caractéristiques principales de la vanne $L G-2$ qui a été conçue par Marine et Neyrpic se retrouvent à quelques détails près sur plusieurs installations importantes au Canada et en France dont la liste est donnée à la figure 2. Elles peuvent se résumer ainsi (voir Fig. 3 relative à $L G \cdot 2)$ :

- la vanne fourreau constitue l'organe de fermeture de sécurité en cas de non fonctionnement du distributeur et le dispositif d'étanchéité lors des périodes d'arrêt de la machine;

- elle est disposée entre l'avant-distributeur et le distributeur et ferme en descendant;

- six servomoteurs hydrauliques placés sur le flasque supérieur commandent la vanne. Ce système est particulièrement simple et robuste ;

- synchronisation très précise assurée par la combinaison d'un système vis-écrou réversible sur chacun des servomoteurs qui transforme la translation du piston en rotation d'un axe permettant d'asservir les servomoteurs entre eux à l'aide de boucles de chaîne ;

- système de mancuvre comportant une sécurité limitant l'effort et une détection de coincement ;

- guidage des tiges de manœuvre au niveau de la bride

\begin{tabular}{|c|c|c|c|c|c|c|c|}
\hline Installation & $\begin{array}{c}\text { Année } \\
(1)\end{array}$ & Quantité & $\begin{array}{c}\text { Puissance } \\
\text { Mw }\end{array}$ & $\begin{array}{c}\text { Chute } \\
\mathrm{m}\end{array}$ & $\begin{array}{c}\text { Diamétre } \\
\text { 1ntérieur } \\
\text { nm }\end{array}$ & $\begin{array}{c}\text { Epaisseur } \\
\mathrm{mm}\end{array}$ & $\begin{array}{c}\text { Hauteur } \\
\text { distrib. } \\
\mathrm{mm}\end{array}$ \\
\hline LA GRANDE 2 (Canada) & 1979 & $8(2)$ & 338,5 & 137,2 & 7592 & 127 & 1390 \\
\hline LA GRANDE 4 (Canada) & 1983 & 9 & 300 & 116,7 & 7400 & 120 & 1376 \\
\hline SARRANS (France) & 1981 & 1 & 63,5 & 84 & 3904 & 85 & 860 \\
\hline SETIENIE DE CANTALES (F) & 1981 & 1 & 38,5 & 62 & 3904 & 85 & 860 \\
\hline L'AIGLE (France) & 1982 & 1 & 133 & 77 & 6000 & 120 & 1332 \\
\hline SAINT GUILLERME (France) & 1982 & 2 & 58 & 275 & 2950 & 100 & 258 \\
\hline
\end{tabular}

(1) Il s'agit de l'année de mise en service du premier groupe lorsqu'il y en a plusieurs.

(2) Parmi les 16 que comporte la centrale

Figure 2 - Tableau des nouvelles réalisations en France et au Canada 


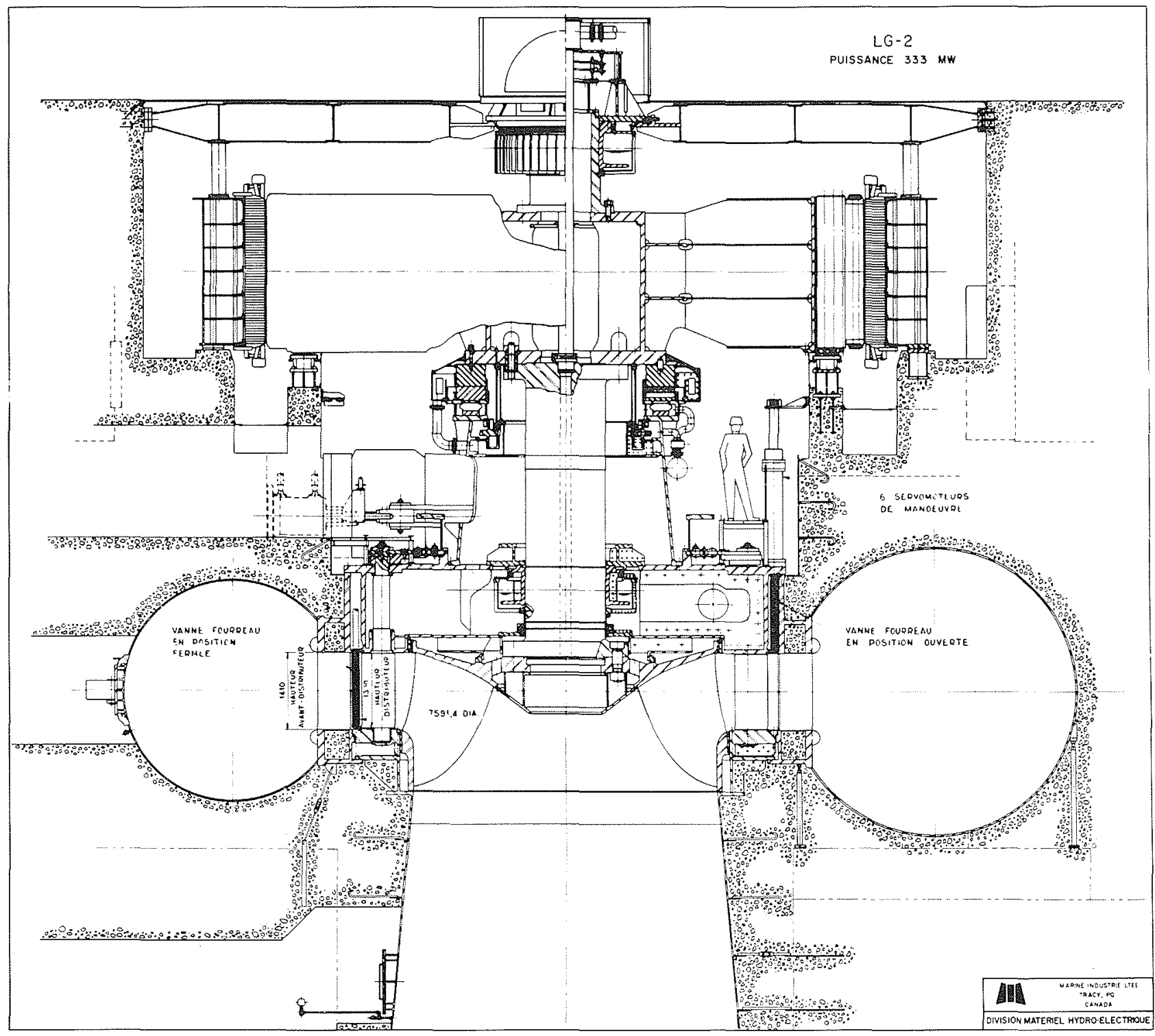

Figure 3 - Ensemble coupe d'un groupe $L G$-2 muni d'une vanne cylindrique

du flasque supérieur et guidage de la vanne avec jeu sur les bords de fuite des avant-directrices;

- fermeture sous l'action de servomoteurs double effet ou sous son propre poids et, avec l'obtention de la résultante des efforts hydrodynamiques vers le bas, les servomoteurs peuvent être à simple effet. Dans ce dernier cas, la vanne est dite autoclave et peut donc fermer sans apport d'énergie externe ;

- positionnement de la vanne parfaitement sûr; à l'ouverture, la vanne est maintenue plaquée par la pression de commande sur la bride du flasque supérieur, à la fermeture, elle est en appui sur le flasque inférieur;

- les joints d'étanchéité de la vanne fermée sont placés sur les parties fixes et situés tant à la partie haute qu'à la partie basse, à l'aval de la vanne, c'est-à-dire sur le diamètre intérieur ;

- les étanchéités sont accessibles, sauf pour les machines où la hauteur du distributeur est insuffisante, depuis l'intérieur du conduit, ce qui les rend facilement démontables.
La figure 4 montre la vanne cylindrique $L G-2$ en cours d'usinage. La vanne est formée à partir de tôles roulées en acier doux. Son diamètre extérieur est de

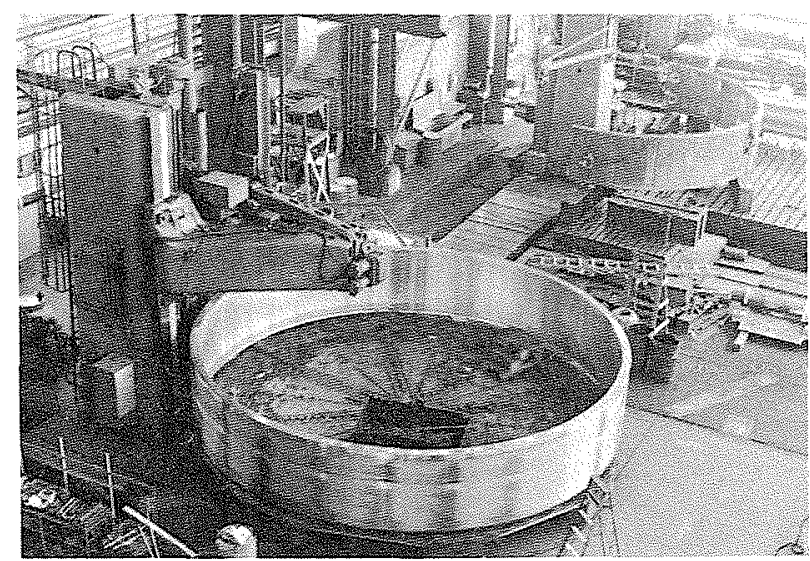

Figure 4 - Vanne fourreau $L G-2$ en cours d'usinage dans les ateliers de Marine Industrie Limitée 
$7846 \mathrm{~mm}$, sa hauteur $1460 \mathrm{~mm}$, son épaisseur $127 \mathrm{~mm}$; elle pèse $35 \mathrm{t}$. Des rechargements en acier inoxydable sont déposés par soudure au droit des joints d'étanchéité supérieur et inférieur et sur les bandes de guidage face aux avant-directrices. Sur les autres parties, un revêtement d'aluminium projeté à chaud avec une épaisseur minimale de $0,1 \mathrm{~mm}$ protège la vanne contre la corrosion en eau stagnante. En raison des limites du gabarit de transport, le fourreau est fabriqué en deux segments qui sont assemblés par des joints de soudure en centrale. Les guides en bronze sont usinés en fonction de la forme de l'avant-distributeur et de la vanne elle-même pour être ensuite soudés aux bords de fuite des avant-directrices (voir Fig. 5). Lorsque la vanne est relevée, elle est complètement hors de l'écoulement. La géométrie de son arête inférieure et du seuil se marie parfaitement aux pièces adjacentes; elle forme un léger convergent et ne présente aucune discontinuité dans le passage hydraulique pouvant causer de la cavitation et des pertes d'énergie (voir Fig. 5 et 6).

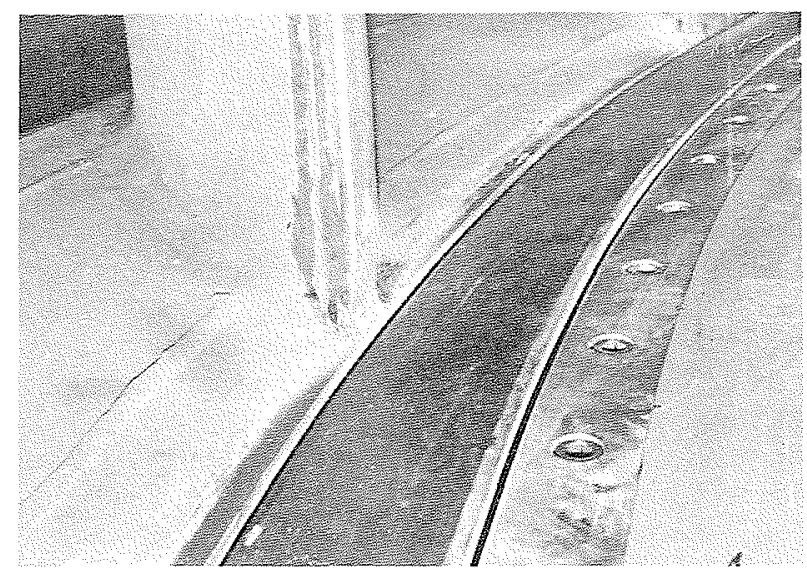

Figure 5 - Arête inférieure de la vanne ouverte et guide en bronze sur avant-directrice à la centrale $L G-2$

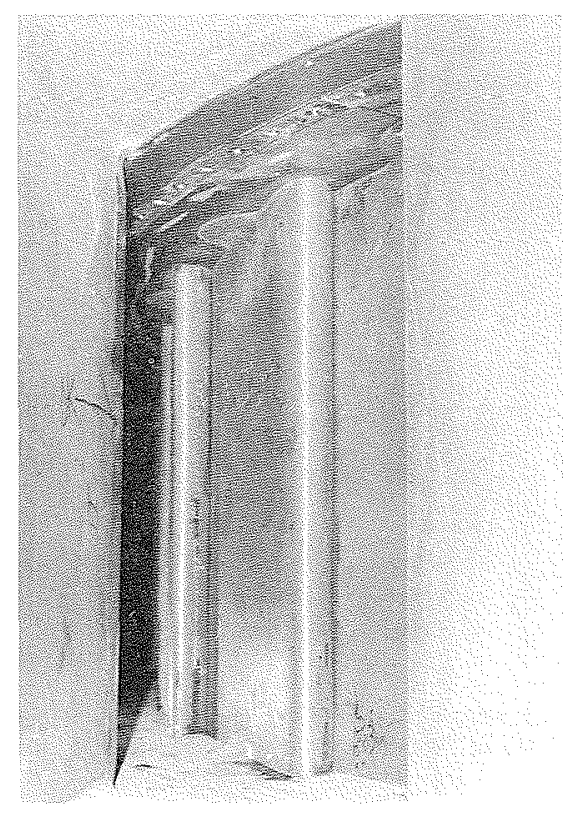

Figure 6 - Vue du distributeur de la bâche montrant la continuité du conduit à l'arête inférieure et au seuil de la vanne $L G-2$.

\section{Essais sur modèles}

Le dimensionnement des vannes fourreau et de leurs organes de commande est basé sur des résultats d'essais sur modèles comportant la turbine et la vanne. A l'occasion notamment des affaires La Grande 2 et La Grande 4 , outre les caractéristiques générales de la turbine, nous avons mesuré pour différentes ouvertures du distributeur et de la vanne, au cours de fonctionnements au synchronisme et à l'emballement :

- les pressions existant de part et d'autre du fourreau et sous le couteau de la vanne;

les fluctuations de pression en divers points de l'écoulement ;

- et, pour déterminer l'interaction de la vanne fourreau sur le distributeur placé directement à l'aval, les couples hydrauliques appliqués sur deux directrices.

Nous donnons figure 7 l'évolution des principaux paramètres observés sur le modèle $L G-4$ pour une course complète de vanne fourreau alors que le distributeur est pleinement ouvert en fonctionnement synchrone. Pour ce régime, la perte de charge varie rapidement au début et à la fin de la course. Les pertes obtenues au synchronisme sont supérieures à celles mesurées à l'emballement sauf pour les faibles ouvertures de vanne et de distributeur où elles deviennent égales. Il est normal d'obtenir ces résultats puisque le débit de la machine à l'emballement est inférieur à celui de la machine au synchronisme.

Les fluctuations de pression mesurées sous la vanne fourreau au droit du seuil et sous la roue atteignent leurs valeurs maximales dans les derniers 20 pour cent de la course. C'est pour la pleine ouverture du distributeur en régime synchrone que les plus grandes valeurs sont rencontrées. On peut voir leur variation en fonction de l'ouverture relative de la vanne sur la figure 7. L'analyse des fluctuations de pression sous la roue a montré qu'aux faibles ouvertures de la vanne, les pulsations sont

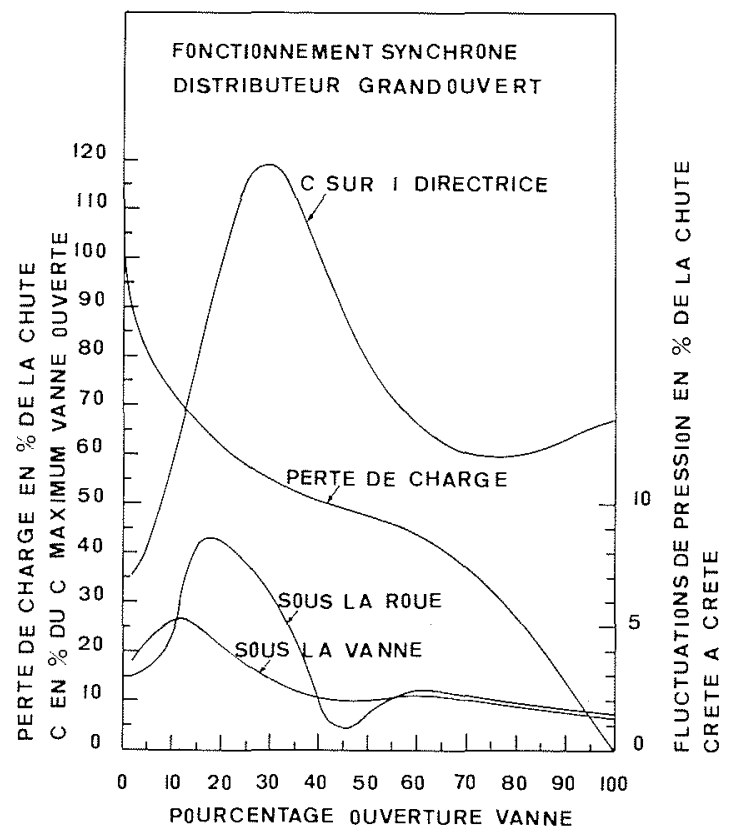

Figure 7 - Evolution des principaux paramètres étudiés sur le modèle $L G-4$ 
rendues maximum par l'action de la vanne elle-même alors que pour les positions proches de la pleine levée c'est le fonctionnement de la roue aux charges partielles qui les crée. A l'emballement, les fluctuations de pression sont fortement tributaires de la roue dont l'influence se fait sentir jusqu'aux faibles ouvertures de la vanne où l'on retrouve des valeurs proches de celles obtenues au synchronisme.

La valeur maximum du couple sur les directrices est atteinte pour la pleine ouverture du distributeur et pour une levée de vanne égale à $30 \%$ environ. Rapportée à la tendance à fermer maximum obtenue avec la vanne complètement ouverte, cette valeur est de 1,19 au synchronisme et 1,13 à l'emballement. Rapportée au couple hydraulique de mancuvre des directrices à pleine ouverture lorsque la vanne se trouve complètement ouverte, elle atteint près du double (Fig. 7). Ce résultat peut s'expliquer par le fait que, bien que les écoulements se limitent à une partie de la hauteur de la directrice, les vitesses y sont beaucoup plus élevées. Par ailleurs, les angles d'injection à l'entrée du distributeur ne sont plus uniquement déterminés par les avant-directrices; l'influence de la vanne devient prépondérante dès qu'elle commence à contrôler le débit. La structure de l'écoulement se trouve de plus perturbée par l'influence de la roue qui provoque des recirculations à la partie haute des directrices derrière la vanne.

Les valeurs obtenues pour les pressions au voisinage de la vanne et pour les couples sur la directrice à différentes ouvertures de la vanne et du distributeur mettent bien en évidence la complexité des écoulements à travers ces deux organes. Ce sont les données recueillies sur modèle qui permettent l'évaluation des efforts hydrauliques de mancuvre de la vanne et des surpressions consécutives aux fermetures.

\section{Mise en service de la vanne $\mathrm{LG}-2$}

Pendant l'automne 1979, MLL mettait en service pour le compte de la SEBJ, deux vannes cylindriques qui sont à notre connaissance les plus importantes au monde tant par leurs dimensions que par le débit qu'elles peuvent couper. Un programme d'essais comportant des mesures avec la vanne maintenue statique dans l'écoulement et pendant les ruptures de débit avait été préparé en vue de cette mise en service. La pression en divers points du conduit de même qu'aux servomoteurs de manœuvre et les couples sur les directrices ont été enregistrés. Le débit a été mesuré par la méthode Winter-Kennedy dont les coefficients avaient été calculés lors de l'essai d'indice de la turbine.

En raison du temps passé en régime fortement perturbé, nous croyons que les essais avec vanne fixe dans l'écoulement constituent le pire test qui puisse être imposé à la vanne cylindrique et aux structures. Pour huit positions de vanne, le distributeur était ouvert par tranche de $20 \%$ jusqu'à $100 \%$ pendant que le groupe était maintenu à vitesse synchrone. Pour ne pas dépasser l'amplitude des vibrations de la ligne d'arbre tolérée au cours de la marche normale du groupe, l'ouverture du distributeur a été limitée à $80 \%$ lorsque la vanne était fermée à $90 \%$. Le niveau du bruit mesuré dans le puits turbine à l'aide d'un sonomètre a augmenté d'environ $5 \mathrm{~dB}$ pendant les essais par rapport au niveau observé au cours de la marche normale. En général, l'intensité sonore augmente avec la progression de la fermeture de la vanne et avec l'ouverture du distributeur.

La figure 8 montre l'évolution du débit en fonction de la position de la vanne pour deux ouvertures du distributeur; on remarque la coincidence des valeurs obtenues sur prototype avec celles mesurées sur modèle. C'est à l'approche du seuil qu'un petit écart commence à se manifester. Il peut être expliqué d'une part par l'imprécision des mesures et d'autre part par l'effet d'échelle important et la difficulté de reproduire sur modèle, les paramètres régissant l'écoulement au droit de la vanne pour les faibles ouvertures. La vanne commence à couper véritablement le débit lorsque la vitesse moyenne de l'eau au droit de celle-ci atteint 20 mètres par seconde environ.

Pendant les ruptures de débit qui ont été exécutées en commande double effet, l'alternateur demeurait couplé au réseau. Au cours de ces manœuvres, aucune vibration anormale n'a été observée. Bien sûr, la création de bruits provoqués par cette grande dissipation d'énergie a été constatée. Le dispositif d'aération axiale de la roue dont le fonctionnement a été observé tout au long de ces coupures de charge a certes contribué à l'apaisement des phénomènes. Les fluctuations de pression mesurées à l'aval de la vanne sous le flasque supérieur n'ont pas eu la même évolution que les bruits. Les amplitudes maximum ont été observées alors que la vanne était à micourse. Au plus fort du bruit observé lorsque la vanne est au voisinage du seuil, elles atteignent 10 à $15 \%$ de la chute. Il est difficile de comparer ces résultats à ceux observés sur d'autres types de vanne. A titre d'information, nous pouvons toutefois citer les valeurs obtenues à l'aval des plus grands robinets papillon au monde, que l'on trouve à la centrale d'Outardes 2, également située au Québec. Ces trois papillons, conçus par Neyrpic et construits par Marine, ont un diamètre de $6,55 \mathrm{~m}$ et sont installés sous $84 \mathrm{~m}$ de chute. Lors des coupures de débit effectuées en automne 1978, les fluctuations de pression maximum mesurées à l'aval de la vanne représentaient

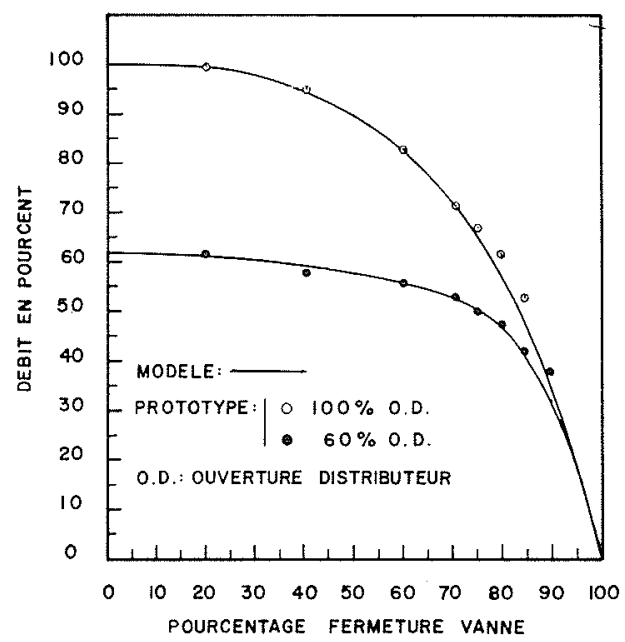

Figure 8 - Evolution du débit en fonction de la position relative de la vanne fourreau $L G-2$. Comparaison des résultats modèle et prototype 


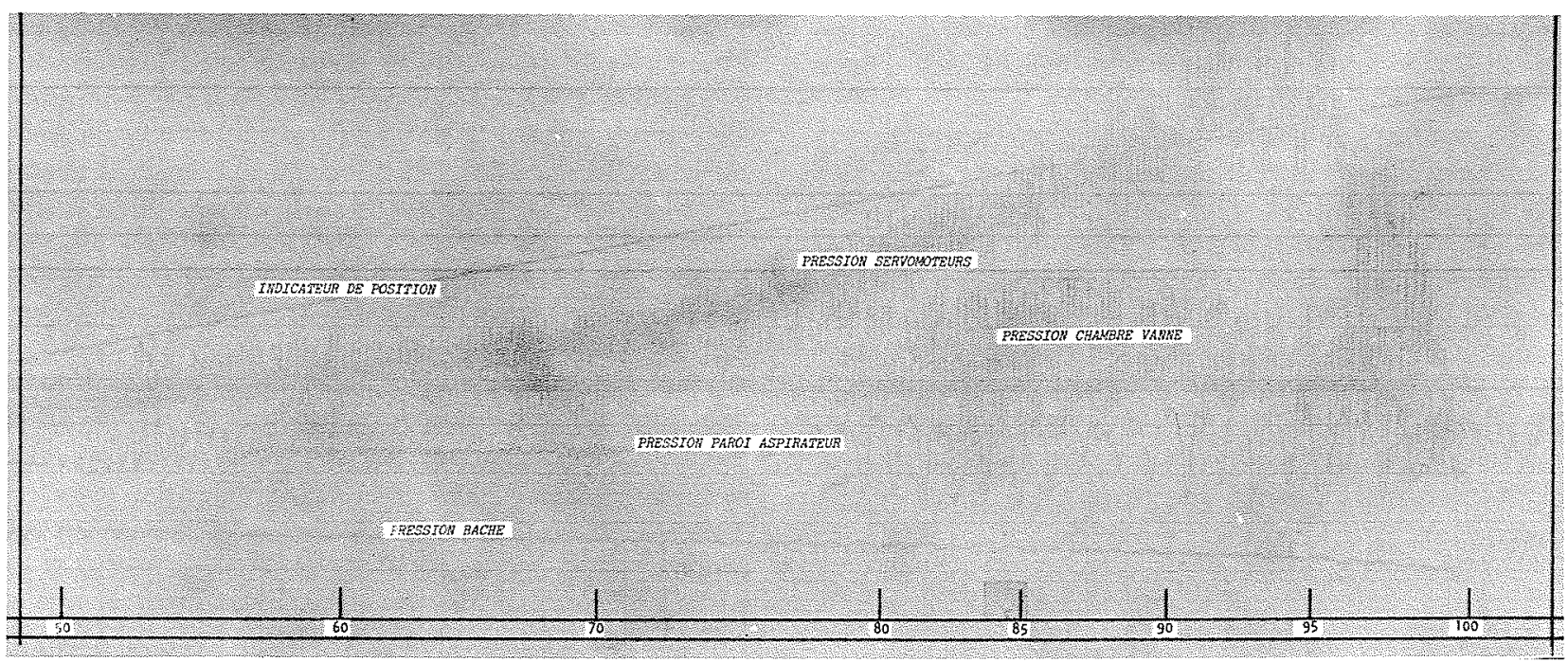

Figure 9 - Extrait d'un enregistrement effectué lors d'une coupure de pleine charge à $L G-2$.

$10 \%$ de la chute. Il est important de souligner que le premier point de mesure se trouvait alors à une distance égale à au moins trois fois le diamètre de l'obturateur.

La figure 9 montre un extrait de l'enregistrement effectué au cours de la coupure de la pleine charge. La surpression relative a été de $15 \%$ pour un temps de manœuvre de 75 secondes. Elle s'est produite à la toute fin de la fermeture. Le signal de l'indicateur de position montre clairement que la fermeture est positive et quasi linéaire en dépit des fluctuations de pression dans la chambre de la vanne qui se répercutent dans le bas des servomoteurs. On remarque la variation caractéristique des efforts de manceuvre au cours de la fermeture. Avant que la vanne ne contrôle véritablement le débit, l'accélération de l'écoulement sous le couteau est insuffisante pour donner lieu à des efforts importants. Avec l'augmentation de la vitesse, la pression sous la vanne diminue jusqu'à produire un décollement et on observe l'accroissement des forces qui atteignent un maximum. En fin de course le convergent formé par l'arête inférieure et le seuil fait augmenter la pression sous la vanne et c'est la tendance inverse qui se manifeste. L'effort hydraulique maximum a été de l'ordre de 300 tonnes. Il s'est produit alors que le distributcur était pleinement ouvert ct la vanne engagée à $90 \%$ dans l'écoulement.

L'évolution de la pression dans la chambre inférieure des servomoteurs de manceuvre en fonction de la position de la vanne fourreau pendant les coupures de débit est montrée à la figure 10 pour deux positions représentatives des directrices. On peut noter sur cette figure que les pressions mesurées ne dépassent pas la frontière supérieure de l'enveloppe établie à partir des résultats d'essais sur modèle. Cette limite avait été obtenue pour la pleine ouverture du distributeur et, si les valeurs mesurées sur le prototype dans ces conditions étaient corrigées pour tenir compte des frottements, elles s'en approcheraient davantage.

En termimant, ajoutons qu'en eau morte lorsque la vanne est complètement appuyée sur ses joints d'étanchéité, aucun phénomène vibratoire n'a été observé dans la conduite d'amenée. De même à pleine ouverture lors-

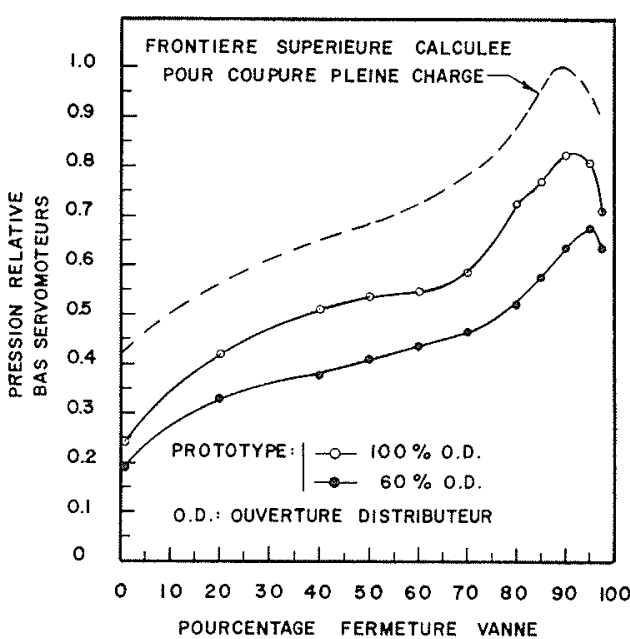

Figure 10 - Evolution de la contrepression dans les servomoteurs en fonction de la position relative de la vanne $L G-2$.

que le groupe est en opération, aucune vibration qui aurait pu être causée par des sollicitations hydrodynamiques n'a été détectée sur la vanne qui est alors fortement plaquée contre la bride du fond supérieur par les mécanismes de mancuvre.

\section{Conclusion}

Le succès rencontré lors de la mise en service des très grandes vannes cylindriques de $L G-2$ et les bonnes expériences d'exploitation en France et au Canada depuis près de vingt ans témoignent des grandes possibilités de ce type d'organe de garde en vue d'applications aux diverses installations hydroélectriques.

L'expérience industrielle acquise grâce aux campagnes de mesures effectuées lors des mises en service et les résultats d'essais sur modèle constituent un bagage précieux qui permet d'envisager avec assurance le dimensionnement de vannes fourreau sur des turbines de 
forte puissance sous des chutes plus élevées pour lesquelles les robinets sphériques atteignent des dimensions et des poids trop importants.

La vanne fourreau est une structure autoportante beaucoup moins complexe que les obturateurs et les corps de papillon ou de sphériques sur lesquels sont concentrées les charges des tourillons. L'absence de vibrations importantes des structures lors des coupures en charge de même que la frabilité des mécanismes de manœuvre et l'efficacité des join ts d'étanchéité devraient servir à dissiper les craintes et les appréhensions à l'égard de ce type de vanne. En définitive, les vannes fourreau ont un bon comportement si leur technologie est robuste et si le système de synchronisation est d'une bonne conception.

\section{Applications nouvelles}

La tendance actuelle est à l'emploi d'installations hydroélectriques prêtes à répondre aux demandes de pointe d'énergie. C'est pourquoi on constate un suréquipement des aménagements pour lequel les avantages présentés par la vanne fourreau méritent d'être considérés.
$\mathrm{Au}$ cours d'essais sur modèle, nous avons constaté que le rendement d'une turbine conserve encore des valeurs acceptables alors que la vanne a déjà fermé notablement. Ce résultat permet d'envisager l'emploi de vannes fourreau sur des machines à distributeur fixe pour partialiser leur puissance, et sur de petites turbines sans distributeur.

Limplantation de vannes fourreau sur des turbines Kaplan, sur des turbines Francis de hautes chutes et sur des turbines-pompes est aussi possible, mais des modifications plus ou moins importantes suivant le cas devront être apportées à la technique actuelle qui a été développée pour des Francis de moyennes chutes. Pour les turbines Kaplan, la grande hauteur du distributeur et la faible hauteur du flasque rendent sa fixation délicate. Sur les turbines Francis de hautes chutes et sur les turbines-pompes, lors des coupures de débit, les grandes vitesses d'écoulement et les régimes fortement perturbés au cours des transitoires mettent en jeu des énergies vibratoires importantes et conduisent à des efforts hydrauliques non axisymétriques pour lesquels la vanne et ses attaches de même que les structures fixes et mobiles devront être soigneusement analysés. Sur ce plan, la réalisation de l'installation, en cours d'étude, de Saint Guillerme sous une chute de $275 \mathrm{~m}$ sera particulièrement intéressante et riche d'enseignements.

\section{Discussion}

M. le Président. - Je remercie M. HUDON qui vient de nous présenter cet exposé pratique mettant en évidence la parfaite cöncidence entre le projet ef la réalisation, notamment en ce qui concerne les efforts, ce qui est in téressant.

$\mathrm{Y}$ a-t-il des questions?

M. LACOSTE. - Je voudrais poser les deux questions suivantes:

- n'y aurait-il pas intérêt à concevoir la vanne de façon à lui conférer une tendance à se fermer sous l'effet de son propre poids. Cela irait dans le sens de la sécurité et permettrait d'obtenir la fermeture même en cas de manque de pression d'huile; - avez-vous réalisé des essais de fermeture avec enregistrement des paramètres principaux en cas de déconjugaison d'une ou plusieurs directrices, par exemple en cas de non fermeture. Connait-on les poussées et frottements qui en résultent et est-on sûr de fermer la vanne en pareil cas?

M. HUDON. - Nous avons travaillé à augmenter la sécurité à la fermeture par l'obtention de l'autoclavité. Le comportement observé à la mise en service des vannes LG-2 nous permet d'être très optimistes quant à cette possibilité. Certaines mises au point sont en cours et nous ferons connaître les résultats lorsque nous aurons recueilli davantage d'informations.

En ce qui concerne votre deuxième question, il est bien certain qu'un essai de ce genre aurait été très intéressant ; mais, à la veille de la pointe de consommation annuelle d'électricité, notre client a préféré les mégawatts aux essais bien qu'il ne se désintéresse pas des problèmes de vanne.

$M$. le Président. - Merci de ces réponses. Je vois que les clients apparaissent partout aussi désagréables mais il leur faut concilier les nécessités d'alimentation de la clientèle en énergie électrique et l'avancement de la technique.

Je pense cependant que cette demande est pertinente, et il sera tout de même bon de contrôler, soit sur votre machine, soit sur d'autres machines de Neyrpic, ces fonctionnements asymétriques, parce que l'expérience montre qu'ils peuvent se produire et qu'il est bon de vérifier les hypothèses de calcul.

M. BOSC. - Je voudrais apporter une lumière nouvelle à votre dernière remarque. Vous avez parlé d'essais à faire avec un certain nombre de directrices désynchronisécs.

Cet essai présente un intérêt particulier pour les vannes de coupure qu'on placerait sur des turbines-pompes. Sur ces machines en effet il se crée, au moment des transitoires, des déséquilibres hydrauliques qui entraînent une dissymétrie importante des efforts.

M. VIRIEUX. - Pour les centrales à plusieurs groupes, le choix de vannes-fourreaux n'implique-t-il pas la présence, à l'amont, de conduites individuelles et ce pour des raisons d'exploitation?

M. HUDON, - Notre client, Hydro-Québec, emploie un peu ce critère. Le personnel hésiterait à aller travailler immédiatement à l'aval d'une vanne tourreau.

Or, comme nous l'avons mentionné,la vanne fourreau est très fiable. Donc, pour le cas où les conduites ne sont pas individuelles, il serait possible d'aménager dans l'avant-distributeur par exemple, une chambre tixe qui permettrait le démontage de composants importants, telles que la roue et les flasques. Par conséquent, le temps d'arrêt d'une série de groupes pourrait être limité aux in terventions sur les bâches seulement.

M. le Président. - Je vous remercie. 